\title{
Effect of added nucleophilic species on the rate of primary amino acid nitrosation
}

\author{
Gabriel da Silva, Eric M. Kennedy* and Bogdan Z. Dlugogorski \\ Process Safety and Environment Protection Research Group, School of \\ Engineering. The University of Newcastle, Callaghan, NSW 2308, \\ Australia.
}

Email: Eric.Kennedy@newcastle.edu.au

Table S1. Equilibrium constants used in the determination of $k_{\mathrm{N}}$ values.

\begin{tabular}{lr}
\hline$K_{\mathrm{ONX}}(\mathrm{ONCl}), \mathrm{M}^{-2}$ & $1.1 \times 10^{-3}$ \\
$K_{\mathrm{ONX}}(\mathrm{ONBr}), \mathrm{M}^{-2}$ & $5.1 \times 10^{-2}$ \\
$K_{\mathrm{ONX}}(\mathrm{ONSCN}), \mathrm{M}^{-2}$ & 32 \\
$K_{\mathrm{ONX}}(\mathrm{ONTU}), \mathrm{M}^{-2}$ & 5000 \\
$K_{\mathrm{ONX}}\left(\mathrm{N}_{2} \mathrm{O}_{3}\right), \mathrm{M}^{-1}$ & $3.0 \times 10^{-3}$ \\
$K_{\mathrm{a}}$ (Alanine $), \mathrm{M}$ & $2.04 \times 10^{-10}$ \\
$K_{\mathrm{a}}$ (Glycine $), \mathrm{M}$ & $2.51 \times 10^{-10}$ \\
$K_{\mathrm{a}}$ (Valine), $\mathrm{M}$ & $2.40 \times 10^{-10}$ \\
\hline
\end{tabular}


Table S2. Product of $k_{\mathrm{N}}, K_{\mathrm{ONX}}$ and $K_{\mathrm{a}}$. For nitrosation by $\mathrm{N}_{2} \mathrm{O}_{3}$, the product is divided by $\left[\mathrm{H}^{+}\right]$, for unit consistency.

\begin{tabular}{cccccc}
\hline & \multicolumn{3}{c}{$k_{\mathrm{N}} K_{\mathrm{ONX}} K_{\mathrm{a}}\left(\mathrm{M}^{-2} \mathrm{~s}^{-1}\right)$} & & $k_{\mathrm{N}} K_{\mathrm{ONX}} K_{\mathrm{a}} /\left[\mathrm{H}^{+}\right]\left(\mathrm{M}^{-2} \mathrm{~s}^{-1}\right)$ \\
& $\mathrm{ONCl}$ & $\mathrm{ONBr}$ & $\mathrm{ONSCN}$ & $\mathrm{ONTU}$ & $\mathrm{N}_{2} \mathrm{O}_{3}{ }^{*}$ \\
\hline Alanine & 0.0011 & 0.025 & 0.48 & 0.81 & 0.0071 \\
Glycine & 0.0022 & 0.037 & 3.0 & 1.2 & 0.022 \\
Valine & 0.0018 & 0.077 & 2.3 & 0.72 & 0.034 \\
\hline
\end{tabular}

* The results for $\mathrm{N}_{2} \mathrm{O}_{3}$ nitrosation cannot be directly compared to the other results, as $\mathrm{N}_{2} \mathrm{O}_{3}$ nitrosation obeys a second order kinetic mechanism (as opposed to first order for the other nucleophiles). However, the values of $k_{\mathrm{N}} K_{\mathrm{ONX}} K_{\mathrm{a}} /\left[\mathrm{H}^{+}\right]$provide an approximate comparison to the $k_{\mathrm{N}} K_{\mathrm{ONX}} K_{\mathrm{a}}$ values.

Table S3. Correlation coefficients $\left(R^{2}\right)$ for Arrhenus activation energy plots.

\begin{tabular}{lccccc}
\hline & $\mathrm{ONCl}$ & $\mathrm{ONBr}$ & $\mathrm{ONSCN}$ & $\mathrm{ONTU}$ & $\mathrm{N}_{2} \mathrm{O}_{3}$ \\
\hline Alanine & 0.994 & 0.955 & 0.988 & 0.983 & 0.937 \\
Glycine & 0.924 & 0.984 & 0.995 & 0.996 & N/A \\
Valine & 0.962 & 0.954 & 0.979 & 0.972 & N/A \\
\hline
\end{tabular}

